$$
\begin{gathered}
I=A s_{1}{ }^{2} \omega_{0} \frac{3}{2 \pi^{2}} \delta \alpha^{2} \mu_{0}{ }^{2} x^{1 / 3}\left[\frac{x^{4 / 3}}{2 y} \Phi_{1}-x^{1 / 3} \Phi_{2}\right], \\
K=A s_{1}{ }^{4} \omega^{2} \frac{\delta \alpha^{2}}{\pi^{2}} \mu_{0}{ }^{2}\left[\Phi_{1}\left(x^{1 / 3}+\frac{3 x^{2}}{4 y}\right)-\frac{2}{3} x \Phi_{2}\right] .
\end{gathered}
$$

Hier haben wir nur im innersten Bereich ein nach Innenströmen der Materie, während weit außen wegen der praktisch starren Rotation sich nichts ändert.
Die zur Herstellung der Abbildungen notwendigen numerischen Rechnungen wurden auf der Göttinger elektronischen Rechenmaschine G 1 durchgeführt, für deren Überlassung ich an dieser Stelle meinen Dank aussprechen möchte.

Meinem verehrten Lehrer, Herrn Professor C. F. v. W e i z . sä c k e r gebührt mein besonderer Dank für vielerlei Hilfe, anhaltende Geduld und wertvolle Anregungen.

\title{
Korngrenzenstruktur und Ladungsträgertransport in Halbleiterkristallen *
}

\author{
Von H. F. MatarÉ * * \\ West End, New Jersey, U.S.A. \\ (Z. Naturforschg. 10a, 640-652 [1955]; eingegangen am 21. Juli 1955)
}

\begin{abstract}
The structural character of boundaries or interfaces between two perfect crystals of different crientation but equal chemical composition defines the behavior of grain boundaries with respect to carrier transport. The amount of misfit in the grain boundary zone, as well as the amount of energy stored by elastic deformation, defines the electrical properties.

The number of free carriers (electrons) in boundary states increases with the cross-potential applied, while positive space charge regions build up on both sides of the boundary. The boundary zone itself has p-type character and becomes more conductive when the number of electrons bound to the dangling bonds increases. Grain boundary zones may be as thick as a few tenths of a mm. Extremely small zones are formed by disturbed twins.

Two and three probe measurements on such bicrystals have been made in order to study the carrier transport phenomena. High current multiplication due to carrier density misfit and gate action in the case of opposite polarization have been found. In addition, contacts were plated to boundary-zones; and modulation through the bulk material, as in a NPN-junction, was studied. Here current multiplication can reach high values even in a base-to-ground connection.

Since those electrons bound to a grain boundary interface by a cross potential may be present only in the form of excitons, in the field of their dangling bonds before adjustment, their time constants for recharging processes might be very short such that it is probable that high frequency response is improved. Basic elements and consequences of the developed theory and the correlation between boundary stress field and carrier transport are outlined.
\end{abstract}

$S^{\text {ent }}$ eit den ersten Arbeiten über das elektrische Verhalten von Korngrenzen durch Pears o ${ }^{6}$, Tay lor und Mitarb. ${ }^{7}$ ist es bekannt, daß „Korngrenzen“ - als solche sind hier die verbindenden Zwischenzonen zwischen zwei Orientierungen (Körnern) verstanden - in N-Typ-Material ganz spezifische Charakteristika haben.

1. Der spezifische Widerstand, quer zur KG-Zone gemessen, springt auf hohe Werte. Zum Beispiel bei

* Nach einem Vortrag, gehalten auf der 55. Sitzung der American Physical Society in New York, 28. Januar 1955. Siehe Literatur ${ }^{1-5}$.

** Forschungslaboratorium der „Tung-Sol Electric Corp.“, Bloomfield, New Jersey, U.S.A.

$* * \%$ „perfekt" wird im Text nur in bezug auf die Kristallstruktur, nicht in bezug auf den chemischen Störstellengehalt angewandt.

1 H. F. M a t a r é, Z. Naturforschg. 9a. 698 [1954].

2 H. F. M a t a ré, H. K ed esdy u. Mitarbb., Phys. Rev. Bull. 30, 40 [1955] u. Phys. Rev. 98, No. 4, 1179 [1955]. einem Germanium-Kristall von $5 \mathrm{Ohm} \cdot \mathrm{cm}$ können Sprünge von $10^{2} \mathrm{bis} 10^{4} \mathrm{Ohm} \cdot \mathrm{cm}$ gemessen werden.

2. Sondenmessungen zeigen, daß das KG-Feld P-Typ-Charakter hat, wenn das perfekte ** Kristallmaterial N-Typ-Charakter aufweist.

3. Der spezifische Widerstand in Richtung der $\mathrm{KG}$-Ebene, parallel zu den freien Bindungen, ist geringer, als der des perfekten Kristalls (vgl. ${ }^{4,5}, 29$ ).

${ }^{3}$ H. F. M a t a r é, Trans., National Convention IRE, New York, März 1955.

${ }^{4}$ H. F. M a t a ré, Techn. Memorandum No. M-1621, Signal Corps Eng. Labs. Project No. 132 A [1955].

5 H. F. M a t a ré, Techn. Memorandum No. M-1647, Signal Corps Eng. Labs. Project No. 132 A [1955].

${ }_{6}$ G. P e a r s o n, Phys. Rev. 76, 459 [1949].

7 W. E. T a y lo r, N. H. O d ell u. H. Y. F a n, Phys. Rev. 88, 867 [1952]. 
Durch diese Arbeiten und weitere experimentelle Befunde $^{8}$ wird der Schỉß nahegelegt, daß Störstellenausscheidung an Korngrenzen von untergeordneter Bedeutung ist, soweit das elektrische Verhalten bei kleinen Verdrehungswinkeln der beiderseitigen Atomgitter in Betracht kommt.

Denn wenn z. B. Kristalle mit N-Typ-Charakter in Richtung auf die KG-Zone erstarrt sind, müßte die KG-Zone im allgemeinen eher eine Anreicherung an Donator-Störstellen aufweisen. In Wirklichkeit ergeben Sondenthessungen stets P-Typ-Charakter der Störzone.

Man kann eine Fülle von Gründen zusammenstellen, die dafür sprechen, daß das außergewöhnliche elektrische Verhalten von KG kleiner Verdrehungswinkel nicht durch Verunreinigungsunterschiede in der Zwischenzone entsteht, sondern daß ausschließlich die freien Bindungen hierfür verantwortlich sind. Einige dieser Gründe seien hier kurz erwähnt ${ }^{9}$ :

1. Die Tatsache, daß KG nur Akzeptorcharakter haben in N-Typ-Material, bei dem der zuletzt erstarrende Teil Donatorcharakter besitzt und in P. Typ-Material elektrisch nicht in Erscheinung treten, spricht dafür, daß die Fehlstellen resp. Leerstellen im Gitterbau ausschlaggebend sind.

2. Mikrophotographien zeigen, zumindest für KG kleiner Winkel, keine Phasenumwandlung.

3. Wird eine KG-Zone mit hohem Widerstandssprung und ihre Umgebung in P-Typ-Material (Akzeptorverunreinigungen) verwandelt, entweder durch Diffusion oder Nukleoneneinstrahlung oder durch Erhitzen und Abschrecken, so verschwindet der KGWiderstandssprung. Er erscheint wieder, wenn das Material durch Erhitzen und langsames Abkühlen zum N-Typ zurückverwandelt wird.

4. In Germanium wurde z. B. Kupferanhäufung für das Verhalten von KG verantwortlich gemacht. Generell kann gesagt werden, daß Abscheidung von Verunreinigungen in $\mathrm{KG}$ erst bei relativ großen Verdrehungswinkeln (vgl. Abb. 1) $\Theta$ eine Rolle spielt ${ }^{10,11}$. Empfindliche Tracer-Methoden zeigen, $\mathrm{da} \beta$ bis in den Bereich $\Theta=10$ bis 15 Grad (Winkelgrad) noch keine nennenswerten Abscheidungen von Verunreinigungen auftreten. Bevorzugte Diffusion in $\mathrm{KG}$ tritt erst von Winkeln über $30 \mathrm{Winkelgrad}$

\footnotetext{
8 W. T a y lor u. K. La rk-H or ovitz, Purdue Semiconductor Research Report, October 1948.

${ }^{9}$ H. F. M a t a ré, Z. Phys., in Vorbereitung.

10 W. R. Thom as u. B. Ch a l mers, Acta Met. 3, 17 [1955].
}

auf, bei denen das Atomgitter schon stark deformiert ist. Solche starken Verwerfungen sind aber vom elektrischen Standpunkt schon uninteressant, da im Diamantgitter diese Winkel leicht zu Zwillingsbildung Anlaß geben und ferner Überlappung der Wellenfunktionen der diskreten KG-Niveaus eintritt.

Im Falle des Kupfers können überdies eine Reihe von Gründen angegeben werden, die eine Bildung von P-Typ-Schichtung durch $\mathrm{Cu}$-Dotierung ausschlieBen ${ }^{9}$.

Der Schluß lag also nahe, daß die Gitterverformung der ausschlaggebende Faktor ist und, wie zu erwarten, Akzeptor-Niveaus in die Störzone einführt.

H. Y. F a n und Mitarb. konnten ferner zeigen, daß unter Polarisation die KG die Eigenschaft hat, in stärkerem Maße freie Ladungsträger (Elektronen) zu binden. Dieses außerordentliche Verhalten der Wachstumsanisotropie in bezug auf den Trägertransport war der Anlaß zur Aufnahme ausgedehnter Untersuchungen an Korngrenzen ${ }^{1}$.

Erste Prinzipien, die durch die Arbeiten aufgestellt werden konnten, lassen sich wie folgt zusammenfassen:

a) Da die Akzeptordichte eine Funktion der Gitterstörung ist und diese wiederum die KG-Energie festlegt, so muß ein Zusammenhang zwischen KGEnergie und elektronischem Verhalten bestehen.

b) Da die Breite der Wachstumsanisotropie und die KG-Energie orientierungsabhängig sind, ist eine außerordentliche Variation der elektronischen Eigenschaften durch Änderung der Orientierung zu beiden Seiten der Bikristallzwischenzone zu erwarten.

c) Da die durch die Gitterdeformierung auftretenden, lokalisierten Wellenfunktionen in diskreten Energie-Niveaus im verbotenen Band hier stark potentialabhängig sind (Auffüllung höherer Niveaus bei Spannungserhöhung), ist geringe Trägheit bei Umladungsprozessen zu erwarten (Frequenzverhalten).

d) Infolge der aufgekrümmten Bänder in der KG-Zone und infolge des Mißverhältnisses der Trägerdichten (hoher Anlagerungsquerschnitt für Elektronen) sind die typischen "Hook“- oder HakenFälle möglich ${ }^{12}$.

11 H. J. G o m berg et al., Final Report P. B. 111 558, United States Department of Commerce, Office of Techn. Serv.

${ }_{12} \mathrm{~W}$. S h o c k ley, Electrons and Holes in Semiconductors. Van Nostrand 1950, p. $68,343$. 
Die folgenden Ausführungen und experimentellen Befunde sollen die Berechtigung der genannten Annahmen stützen und interessante Ausblicke auf das elektronische Verhalten von Bikristallzwischenschichten geben.

\section{Korngrenzentypen und mechanische KG-Spannungsfelder}

Betrachten wir gemäß Abb. 1 ein Korngrenzenfeld. Es sei zur Vereinfachung z. B. die (100)-Ebene eines kubischen Gitters dargestellt. Die beiden Körner rechts und links der KG-Ebene, welche z. B. in die (001)-Ebene fällt, sind um je $\Theta / 2$ verdreht. Die Orientierung $\Phi$ der KG in der zur KG-Ebene senkrechten $x$-Richtung ist zu $45^{\circ}$ angenommen. Die Folge der Verdrehung ist, außer einer Anzahl blokkierter Gitterpunkte („locked lattice points“), eine elastische Verformung des Gitters in einer gewissen KG-Zone 4 . Wie wir weiter unten sehen werden, sind diese beiden Faktoren bestimmend für die Energie der Korngrenze.

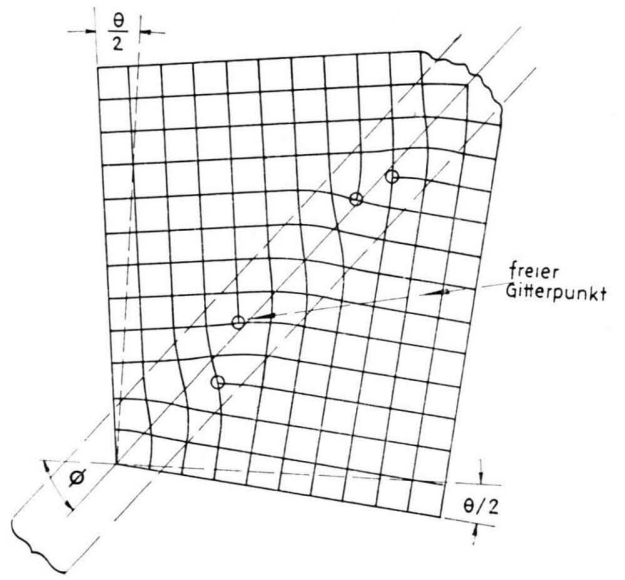

Abb. 1. Schematische Darstellung einer Korngrenze. (100). Ebene eines kubischen Gitters in der Zeichenebene. Die (001). Ebene enthält die KG-Ebene; $\Theta \mathrm{KG}$-Winkel, $\Phi$ KG-Orientierungswinkel, $\triangle$ KG-Zonenbreite, einschließlich elastischer Deformierung.

Messungen von KG-Energien als Funktion des Verwerfungswinkels sind bereits durchgeführt worden ${ }^{13}$. Theoretische Überlegungen zur $\Theta$-Abhängigkeit der KG-Energie ergaben erstaunlich gute Übereinstimmung mit den Meßresultaten ${ }^{14,15}$.

13 C. G. Dunn u. F. L i o n e t t i, Trans. Amer. Inst. Min. Metall. Engrs. 185, 125 [1949].

14 W. T. R e a d u. W. S h o c k le y, Phys. Rev. 78, 275 [1950].
Maximalwerte der Energie liegen zwischen $\Theta=15^{\circ}$ und $\Theta=35^{\circ}$. Für Siliciumcarbid, das wegen seiner Struktur (hexagonal) interessiert, ergibt sich

$$
\Theta_{\max }=30^{\circ} \text {. }
$$

Die von Read und Shockley ${ }^{14}$ abgeleitete Beziehung für die Energie der KG als Funktion von $\Theta$ lautet

$$
E=E_{0} \Theta A+E_{0} \Theta \ln (1 / \Theta) ;
$$

$E_{0}$ ist hier gegeben durch

$$
E_{0}=G a(\cos \Phi+\sin \Phi) / 4 \pi(1-\sigma) .
$$

Die Bezeichnungen sind: $G$ Elastizitätsmodul des Kristalls, $a$ Gitterkonstante, $\sigma$ Poissonsche Konstante, $\Phi$ Orientierung.

$A$ ist ebenfalls eine Funktion der KG-Orientierung. In den meisten Fällen genügt es, zu setzen

$$
A=\text { const }=0,3 \text {. }
$$

Für $\Phi=90^{\circ}$ erhalten wir

$$
E_{0}=G a / 4 \pi(1-\sigma) .
$$

Die Beziehung $E=f(\Theta)$ und die normierte Form nach Read ist schematisch in Abb. 2 dargestellt.
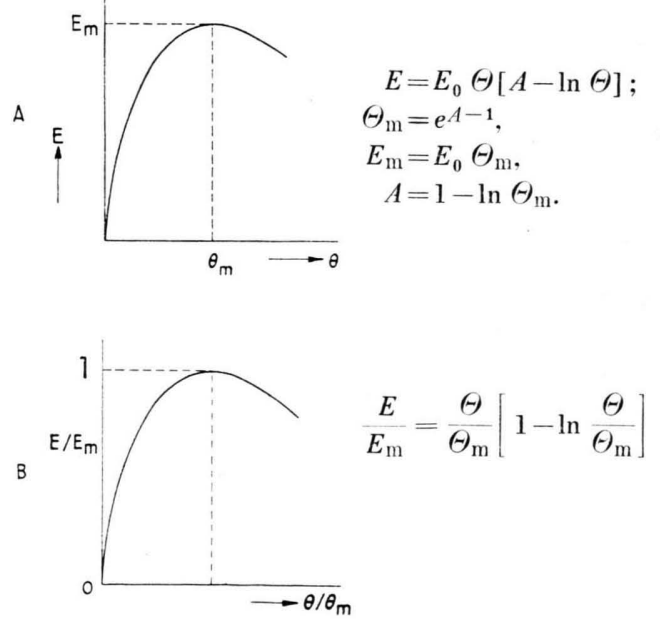

Abb. 2. Korngrenzenenergie als Funktion des Verformungswinkels $\Theta$ (nach Read-Shockley) (A). Relative KG-Energie $E / E_{\max }$ als Funktion der relativen Verformung $\Theta / \Theta_{\max }(\mathrm{B})$.

Die Ableitung dieser Beziehungen kann auf dreierlei Weisen erfolgen:

1. Man bildet das Volumenintegral der Spannungsenergiedichte über den ganzen Körper. 1953.

15 W. T. R e a d, Dislocations in Crystals, McGraw Hill 
2. Man integriert die Arbeit, welche zur Erzeugung des Spannungszustandes durch Oberflächenkräfte notwendig ist, über die gesamte KG-Zone, inklusive der Oberflächendiskontinuität (Gleitebenen).

3. Man bestimmt die Arbeit, die zur Erzeugung der relativen Verschiebungen gegen vorhandene Anziehungs- und Abstoßungskräfte notwendig ist.

Die Methode nach 1. wurde von $R$ ead und $\mathrm{Sh} \mathrm{ock} \mathrm{le} \mathrm{y}{ }^{14}$ angewandt. Das Verhalten in mechanischer Hinsicht von Strukturinhomogenitäten, insbesondere vom Korngrenzentyp, ist in der Literatur vielfach behandelt ${ }^{16-20}$.

Die elektrischen Eigenschaften solcher Zonen beginnen aber erst in letzter Zeit Interesse zu wecken.

Shockley ${ }^{21}$ machte den bedeutsamen Vorschlag, den durch freie Gitterpunkte z. B. einer Kantenverschiebung (edge dislocation) entstehenden Reihen von Akzeptorzentren Reihen von Wellenfunktionen im verbotenen Band zuzuordnen.

Wenn diese Niveaus teilweise gefüllt sind, kann man solche Verschiebungszentren als ein eindimensionales, entartetes Elektronengas auffassen. In Abhängigkeit vom Leitungscharakter sind diese Korngrenzen auch als „Oberflächenzustände“ aufzufassen. $\mathrm{R}$ e a d ${ }^{22,23}$ stellt demgegenüber ein Modell mit nur einem, definierten Niveau unmittelbar unterhalb des Fermi-Niveaus auf. Untersuchungen an Bikristallen definierter Orientierung scheinen zugunsten des Shockleyschen Modells zu entscheiden. Messungen an KG mit einer Verdrehung: $\Theta_{1}=5^{\circ}, \Theta_{2}=0^{\circ}$, $\Theta_{3}=0^{\circ}$ und $\Phi=90^{\circ}$ zeigen, da $\beta$ Polarisation quer zur KG die bekannten Widerstandssprünge ergibt, die auf dem Sperrschichtcharakter der KG-Zone beruhen, daß aber Polarisation parallel zur KG-Ebene einen deutlichen Abfall der $\varrho$-Werte ergibt ( siehe $^{9}$ ). Wie stark diese Leitfähigkeitszunahme parallel zur KG ist, hängt von der Orientierung der freien Gitterpunkte ab und von der Richtung, in welcher die Spannung angelegt wird.

Der Effekt sollte groß sein, wenn die Gitterverschiebung so regelmäßig ist, daß die freien Gitterpunkte alle in einer Ebene liegen. Dann treten hohe Widerstandswerte im rechten Winkel zu dieser Ebene

16 H. E. B u c k l e y, Crystal Growth, John Wiley \& Sons, New York 1952, p. 299.

17 A. R. V e r m a, Crystal Growth and Dislocations, Butterworth Scientif. Publications, London 1953.

18 A. H. Cottrell, Dislocations and Plastic Flow in Crystals, Oxford Clarendon Press, 1953.

${ }_{19}$ W. Shockley, J. H. Hollom a n, R. M a u e r e r auf und niedrige Werte parallel zu den sich überlappenden Coulomb-Feldern der einzelnen, freien Bindungen, die, wenn sie mit Elektronen besetzt sind, eine negative Linienladung darstellen.

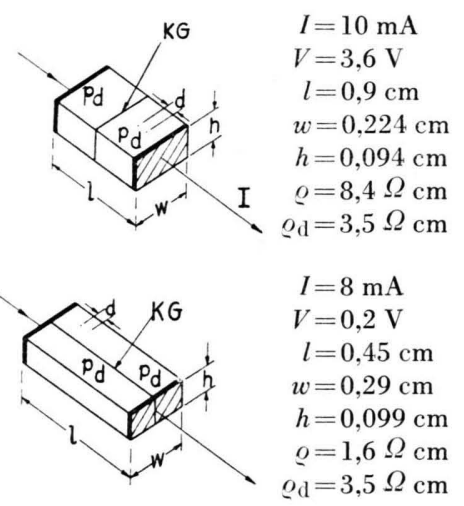

Abb. 3. 1. Messung des spezifischen Widerstandes an Bikristallen, quer zur KG. 2. Dasselbe parallel zur KG-Ebene. (In der Abb. lies $\varrho_{\mathrm{d}}$ statt $p_{\mathrm{d}}$.)

Abb. 3 erläutert eine Messung an Bikristallen. Im ersten Falle wurde senkrecht zur KG-Ebene polarisiert, im zweiten Falle in Richtung der KG. Wie zu erwarten, tritt der Beitrag der KG-Ebene im ersten Falle dadurch zu Tage, daß ein spezifischer Widerstand von $8,4 \mathrm{Ohm} \cdot \mathrm{cm}$ gemessen wurde, im zweiten Falle wurden nur $1,6 \mathrm{Ohm} \cdot \mathrm{cm}$ festgestellt, während das Material außerhalb der KG auf beiden Bikristallen einen spezifischen Widerstand von $3,5 \mathrm{Ohm} \cdot \mathrm{cm}$ aufwies. Dies ist nur denkbar, wenn die erwähnte Ausbildung von Reihen von Energieniveaus im verbotenen Band auftritt. Im 2. Fall, Abb. 3, tritt also eine Konzentration der Stromlinien entlang der KGEbene auf, wie dies auch in Sondenmessungen bestätigt wurde ${ }^{9}$.

Wis Formel (1) für die KG-Energie zeigt, sind zwei wesentliche Anteile vorhanden: $E_{0} \Theta A$ ist der Anteil je freier Bindung, $E_{0} \Theta \ln (1 / \Theta)$ ist repräsentativ für die elastische Gitterenergie.

Wenn $\Theta$ den Optimalwert übersteigt, tritt Überlappung der diskreten Wellenfunktionen auf, was dann zu einer Abnahme der Energie führt. Insofern ist $E$ nicht einfach proportional zur Anzahl der im

u. F. S e itz, Imperfections in nearly perfect Crystals, J. Wiley \& Sons, New York 1952.

20 C. S. B a r r e t, Structure of Metals, McGraw Hill, New York 1952, p. 393.

21 W. S h o ckley, Phys. Rev. 91. 228 [1953].

22 W. T. Re a d, Jr., Pril. Mag. 45, 775 [1954].

${ }_{23}$ W. T. R e a d, Jr., Phil. Mag. 45, 1119 [1954]. 
Gitter eingeführten Akzeptorterme, und anderseits ist das elektrische Verhalten sicher eher durch $E$, als durch die Anzahl der freien Valenzen in der KG. Zone bestimmt. Die Lage im Bandschema von solchen Elektronen, die an als Akzeptoren wirkende Gitterlücken angelagert sind, muß etwas unter dem FermiNiveau angenommen werden ${ }^{24}$.

Vielfach werden die KG-Akzeptorterme besetzt sein (besonders bei angelegter Querspannung), so daß dann dieser Term als Löcherfalle wirksam ist. Da eine Reihe solcher Termlagen unterhalb des Fermi-Niveaus im N-Typ-Halbleiter anzunehmen ist und außerdem mehrere Umladungen wahrscheinlich sind, haben wir hier den Fall von Cis-Akzeptoren (zur Terminologie siehe Schottky ${ }^{25}$ ) vor uns, die nach Aufnahme eines Elektrons zu "TransDonatoren“ werden, was besagt, daß diese Terme dann nahe dem Leitungsband und etwas über dem Fermi-Niveau liegen. Es ist denkbar, daß so auch gerade im Falle der Korngrenzen Cis-Donatoren und Trans-Akzeptoren entstehen. Eher wahrscheinlich ist wohl aber eine Lage nahe der Mitte des verbotenen Bandes, womit bei KG besonders Medial-Donatoren und Medial-Akzeptoren bei der Wiedervereinigung und Entstehung von Paaren wirksam sind. Die Anzahl der Akzeptorterme muß im Gleichgewichtsfall durch die Zahl der freien Gitterbindungen gegeben sein, also zwischen $N=10^{14} / \mathrm{cm}^{3}$ und $10^{16} / \mathrm{cm}^{3}$ schwanken. Im Falle der KG unter Spannung können sicher wesentlich höhere Werte auftreten.

Pears on et al. ${ }^{26}$ haben durch Messungen der Beweglichkeit an mechanisch deformierten Ge-Einkristallen gezeigt, daß Deformierung nur im Falle von N-Typ-Material starken Einfluß auf die Beweglichkeit der Ladungsträger hat. Damit wird der Akzeptorcharakter der Gitterdefekte in KG-Zonen deutlich: Nur im Falle des N-Typ-Kristalls können die an die Akzeptorzentren gebundenen Elektronen die zur Stabilisierung notwendige, positive Raumladungszone aufbauen. Infolge der festeren Bindung des unabgesättigten Elektrons der „freien“ Gitterpunkte würde auch im P-Typ-Material zunächst die Tendenz zur Anlagerung von Elektronen in der KG-Zone bestehen. Es könnten sich aber hier nicht die zur Stabilisierung des Zustandes notwendigen positiven Raumladungszylinder aufbauen.

${ }^{24}$ F. L. Vo g e l, W. T. R e a d u. L. C. Love 11 , Phys. Rev. 94, 1791 [1954].

25 W. S c h o t t k y, Halbleiterprobleme, Vieweg u. Sohn, Braunschweig 1954, p. $85 \mathrm{ff}$.
Da also die freien Bindungen einer KG-Zone als Akzeptorzentren negative Ladung binden und als „Fallen“ wirksam sind, ist ihr starker Einfluß im Sinne einer Beweglichkeits- und Lebensdauer-Verminderung der Minoritätsladungsträger (Löcher) verständlich ${ }^{26}$.

Generelle Regeln, diese Zonen betreffend, welche auf Grund der bisherigen Messungen aufgestellt werden können, sind:

a) Zwillingsgrenzen zwischen Kristallen haben neben geringster Ausdehnung auch geringste Energie und geringste Ausbildung einer Doppelschicht. (Beim idealen Zwilling keine Störung der Valenzbindungen.) Nur gestörte Zwillinge („Franck- oder Shockley-Partials“) oder solche mit gleitender Symmetrieachse (siehe Abb. 4) haben höhere KG-Energie und Doppelschicht. Zwillingsbildung bei Erstarrungsprozessen (zyklische Zwillinge, siehe z. B. Abb. $5^{*}$ ) führt meist zu KG geringerer Energie und gering ausgebildeter Doppelschicht.

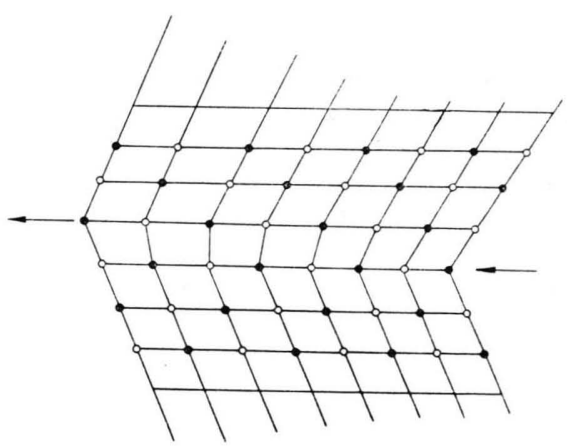

Abb. 4. Schema eines gestörten Zwillings mit gleitender Symmetrieachse.

b) Die KG-Energie ist korreliert zur Höhe der Widerstandssprünge einerseits und zur Fallenwirkung andererseits. Für sehr kleine Winkel $\Theta$ kann die KG oft nur als leichte Schwankung des spezifischen Widerstandes festgestellt werden ${ }^{27}\left(\Theta \leqq 1^{\circ}\right)$. Wenn $\Theta \geqq \Theta_{\max }$ (für den jeweiligen Kristall; meist $30^{\circ}$ ), tritt Úberlappung der Wellenfunktionen der isolierten Bindungen auf und damit Abnahme der Doppelschichtwirkung.

c) N-Typ-Material mittleren spezifischen Widerstandes zeigt maximale, elektrische Effekte. Silicium mit geringer P-Leitung scheint ebenfalls hohe KG-

26 G. L.P e arson, W. T. Read u. F. J. Morin, Phys. Rev. 93, 666 [1954].

* Abb. 5, 7, 8, 9 u. 12 auf Tafel S. 644 a und 644 b.

${ }^{27}$ G. Z i e l a s e k, Z. Metallkunde 46, H. 3 [1955]. 


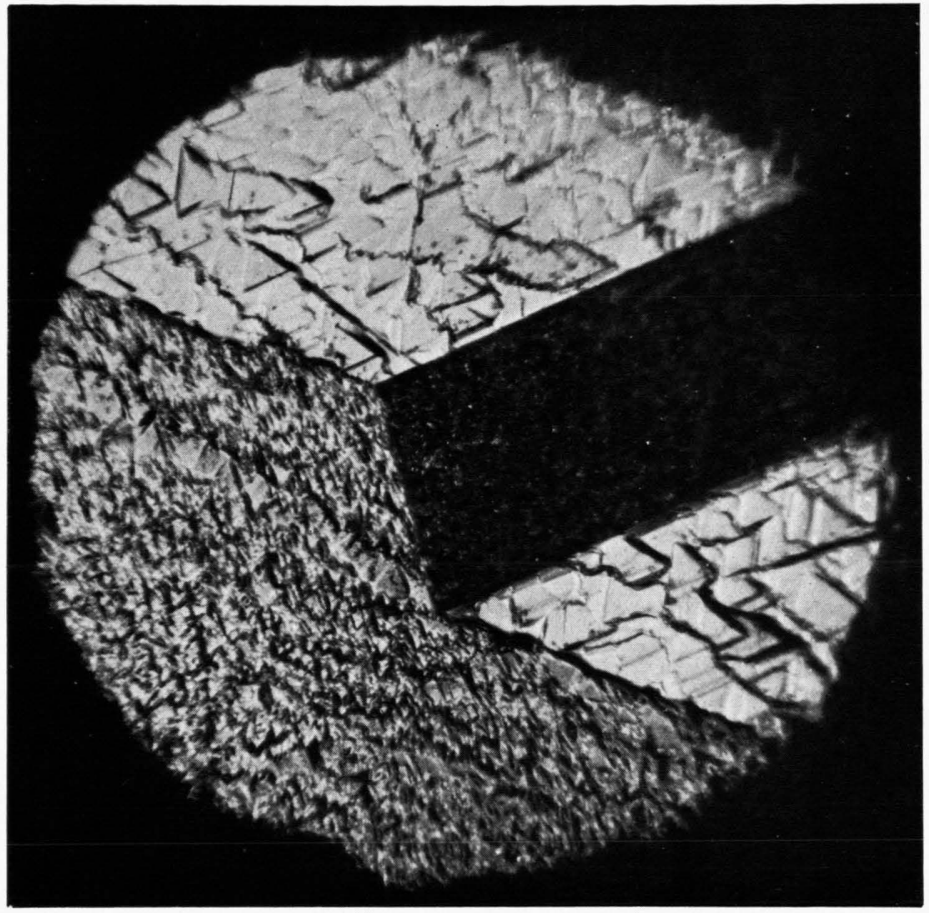

Abb. 5. Mikroaufnahme eines Ge-Kristalls mit typischer Zwillingsbildung. (Zyklische Zwillinge.)

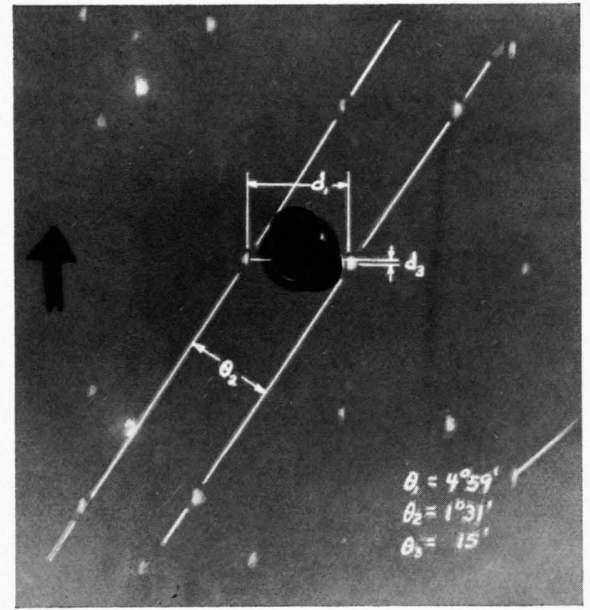

Abb. 7. Laue-Aufnahme eines Germanium-Bikristalls (15 $\mathrm{Ohm} \cdot \mathrm{cm}, \mathrm{N}-\mathrm{Typ})$ mit Entnahme der Verwerfungswinkel. D : Kristall C; : Kristall D

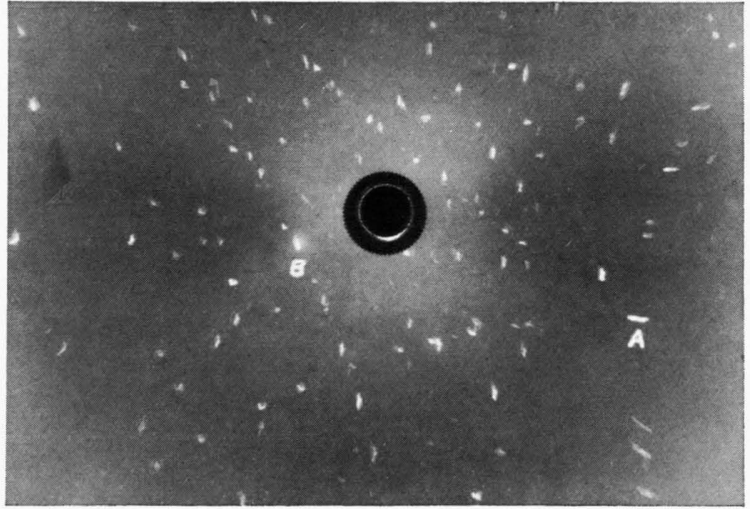

Abb. 8. Laue-Bild eines Silicium-Bikristalls (5 $\mathrm{Ohm} \cdot \mathrm{cm}$, N-Typ). Hauptverwerfungswinkel $\Theta_{1}=37^{\circ}$. A, B : Symmetriezentren. 

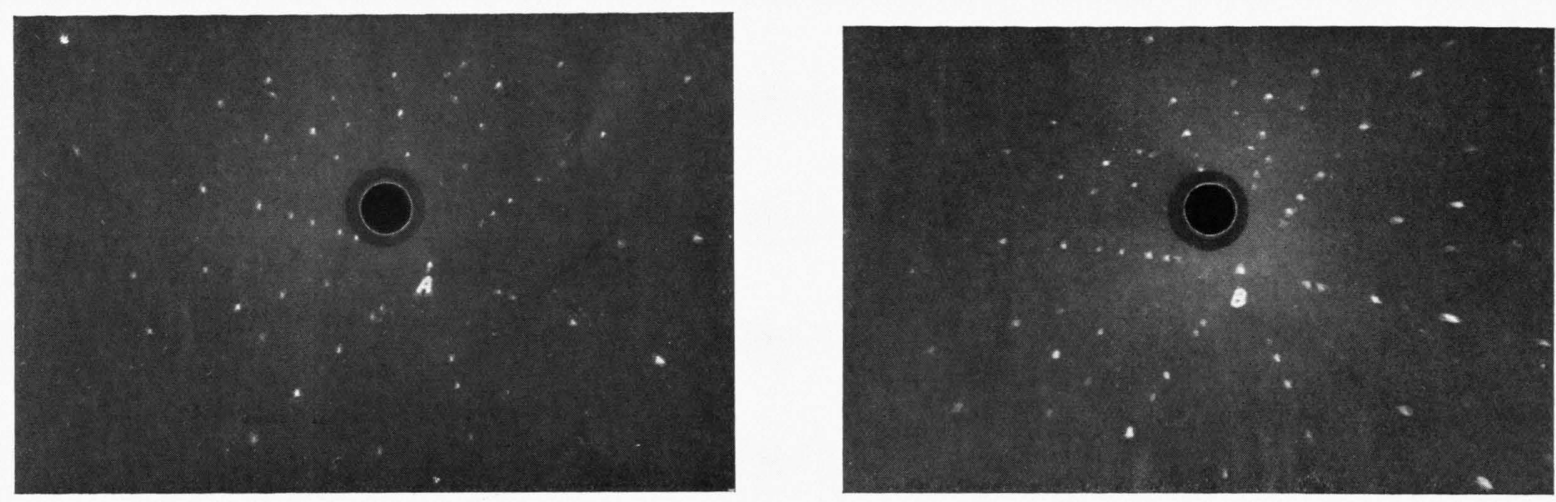

Abb. 9. Wie Abb. 8, jedoch für extrem große Verformung, $\Theta_{1}=40,1^{\circ}$. Die beiden anderen Winkel nahe $0^{\circ}$.

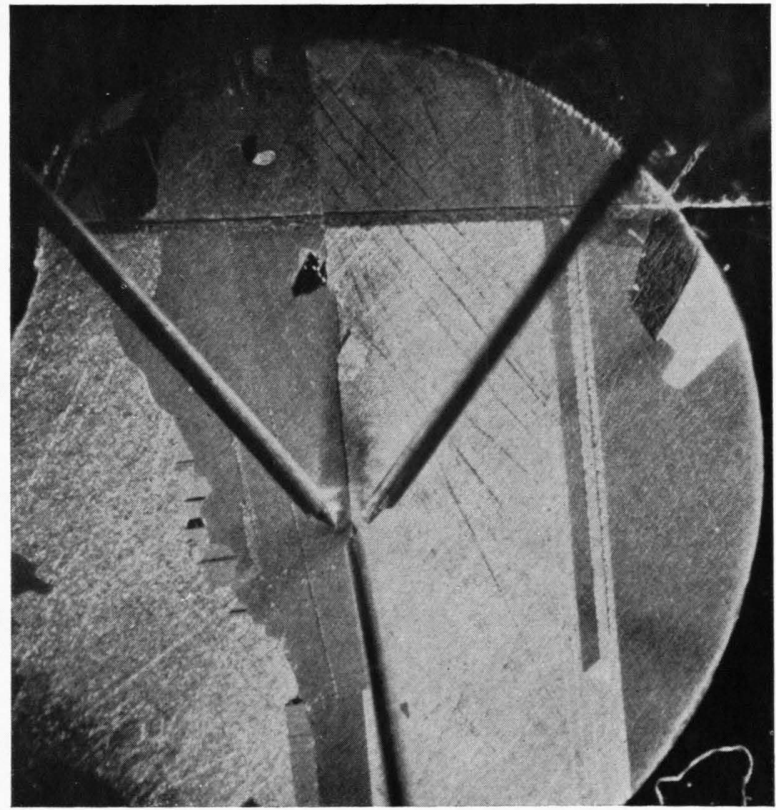

Abb. 12. Dreisondenanordnung auf einer KG in polykristallinem Silicium.

$$
1 \mathrm{~mm}
$$


Sperrschichten aufzubauen, wenn durch entsprechende Polarisation die Defektelektronen aus dem der KG anliegenden Material verdrängt werden. Ist $n_{i} \mathrm{zu}$ klein (z. B. $10^{14} \mathrm{~cm}^{-3}$ ), so steigen die spezifischen Widerstandswerte so stark an, daß der Einfluß des Widerstandssprunges in der KG-Zone verschwindet. Anderseits wird zu hohe Störstellendichte im Sinne einer Überkompensation der durch die KG eingeführten Akzeptorterme wirken.

\section{Herstellung von Bikristallen}

Die definierte Form von Korngrenzen, bei denen die Zone kristallographischer Störung symmetrisch ausgebildet ist und die freien Gitterpunkte in glatter Front das sonst perfekte Kristallmaterial durchqueren, kann, wie sich gezeigt hat, nur in einer präzise gebauten Kristallziehapparatur moderner Form unter Benutzung genau eingestellter Saatkristallhalter $(1 / 4 \mathrm{Grad}$ genau $)$ realisiert werden. Gewisse Schwierigkeiten treten bei der Herstellung von Verdrehungswinkeln $\Theta_{1} \leqq 1^{\circ}$ auf. Da nämlich $\Theta$ drei Freiheitsgrade hat, müssen bei definierten Verhältnissen die beiden anderen Winkel in guter relativer Genauigkeit zu $\Theta_{1}$ bei Null liegen. Die Toleranzen für Saathalter und Ziehapparatur (Temperaturgradient!) werden dadurch extrem und kaum erfüllbar.

Im allgemeinen ist es aber relativ einfach, $\mathrm{KG}$ mit größeren Winkelabweichungen herzustellen. Abb. 6a stellt eine Saatkristallanordnung mit Bikristallblock dar. $\Theta_{1}$ ist der zumeist eingestellte Öffnungswinkel. $\Theta_{2}$ und $\Theta_{3}$ werden dabei annähernd zu Null eingestellt. Abb. $6 \mathrm{~b}$ und c zeigen die Anordnung für die Laue-Analyse des Bikristalls. Ist die Hauptwachstumsrichtung der beiden Kristallseiten bekannt, so liefern zwei Symmetriezentren gleich den Neigungswinkel der Bikristalle, da sie direkte Reflexion des Hauptstrahls sind. Meist erscheinen die Gitterpunkte der beiden Kristallseiten D und C in verschiedener Form, so daß es einfach ist, Verbindungslinien zugehöriger Gitterpunkte zu ziehen und die Winkel $\Theta_{1,2,3}$ aus der einfachen, angegebenen Tangensbeziehung abzulesen ${ }^{28}$. $\Theta_{2}$ folgt direkt aus der Neigung der Geraden durch die beiden Folgen zugehöriger Gitterpunkte zueinander (Abb. 7).

Zwei weitere Aufnahmen von Silicium-Bikristallen mit Angabe der Hauptverdrehungswinkel sind in Abb. 8 und 9 dargestellt.

${ }_{28}$ H. K e d e s d y, American Metallurgist 39, 750 [1954]. a)

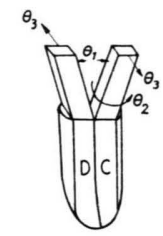

b)

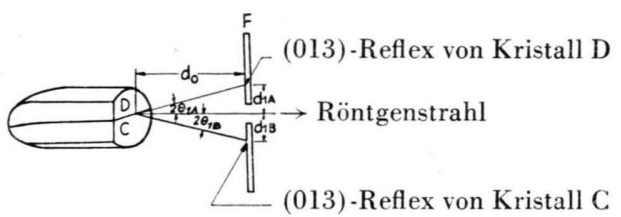

c)

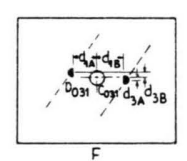

Abb. 6. a) Saatkristallanordnung für Bikristalle mit Angabe der auftretenden Winkel. b) Reflexionsverhältnisse am Bikristall $\mathrm{C}-\mathrm{D}$. Photoplatte $\mathrm{F}$ im Abstand $d_{0}$ von BikristallFläche. c) Trigonometrische Beziehungen für Berechnung der Abweichungswinkel aus der Lage der Reflexe auf dem LaueBild.

Die Symmetriezentren A und B der Bikristallhälften sind angegeben. Bei diesem Material waren die Verdrehungswinkel zu groß (über $30^{\circ}$ ), und die resultierende $\mathrm{KG}$ hatte im ersten Falle Zwillingseigenschaften, im zweiten Falle ebenfalls nur eine gering ausgeprägte Doppelschicht, während der Fall Abb. 7 den oben genannten, extrem hohen Widerstandssprung (Größenordnung $10^{4} \mathrm{im}$ spez. Widerstand ausgedrückt) zeigte.

Weitere Arbeit ist auf dem Gebiet der definierten KG-Herstellung zu leisten, um P-Typ-Zonen solcher Art mit gleicher Sicherheit und gleich steilen Übergängen zu beiden Seiten der Inversionsschicht herzustellen, wie es heute in der Transistortechnik durch reine Verunreinigungsbeladung der Kristalle geschieht.

\section{Korngrenzen-Sperrschichten}

Das elektrische Verhalten von KG ist vergleichbar demjenigen einer Doppelschicht. Nach Arbeiten von $\mathrm{Pears}$ on ${ }^{6}, \mathrm{~T}$ a y lo r ${ }^{8}, \mathrm{M}_{\text {a t a }} \mathrm{e}^{1,4}$ u. a. ist das Verhalten einer KG-Zone vergleichbar demjenigen einer Diode in Sperrichtung, und zwar symmetrisch in beiden Stromrichtungen. Dies natürlich, da eine $\mathrm{N}-\mathrm{P}-\mathrm{N}$-Schichtung zwei mit korrespondierenden Elektroden gegeneinander geschalteten Dioden entspricht. 
Abb. 10 stellt eine solche Kennlinie einer KG in einem Siliciumkristall dar, gemessen zwischen zwei Sonden rechts und links der KG.

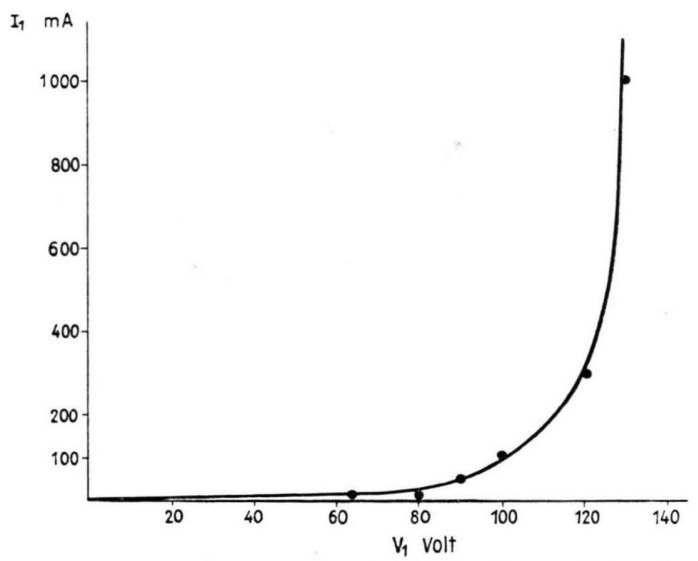

Abb. 10. Strom-Spannungscharakteristik einer Silicium-Korngrenze. Messung zwischen zwei quer zur KG angeordneten Sonden.

Berechnung des KG-Potentials unter Annahme konstanter Elektronenkonzentration in der KG-Ebene:

Wir nehmen an, daß die Bandaufkrümmung an einer polarisierten KG der elektrischen Feldstärke $E$ und dem Potential $V$ über die KG entspreche. Wir gehen aus von dem Ausdruck für die Energiedichte in einem Medium mit verteilten Punktladungen und der Dielektrizitätskonstante $k$ :

$$
\mu=k \int_{\tau} \int_{0}^{E} E \mathrm{~d} E \mathrm{~d} \tau=\frac{k}{2} \int_{\tau} E^{2} \mathrm{~d} \tau,
$$

wo $\mathrm{d} \tau=$ Volumenelement, $V=$ Potential, $E=$ Feldstärke $=$ $-\operatorname{grad} V$.

Nun gilt ferner für den Fall von Punktladungen, $\varrho=e n$, gleichmäßiger Belegung der Poissonsche Satz:

$$
\operatorname{div} E=4 \pi Q=4 \pi \text { e } n \quad(n=\text { Störstellendichte). }
$$

(Annahme vollständig ionisierter Störstellen und kein Feldbeitrag der freien Ladungen.)

Wendet man auf Gl. (4) den Gaußschen Satz an:

$$
\mu=\frac{k}{2} \int_{\tau} E \operatorname{grad} V \mathrm{~d} \tau=-\frac{k}{2} \int_{\tau} V \operatorname{div} E \mathrm{~d} \tau,
$$

so ergibt sich für die Energiedichte andererseits

$$
\mu=\int_{\tau} 4 \pi \varrho V \mathrm{~d} \tau,
$$

und man erhält daher gemäß (4) und (5)

$$
V=k E^{2} / 8 \pi \text { en }
$$

als Ausdruck für das KG-Potential. Für verschiedene Störstellendichten und Felder zu beiden KG-Seiten gilt

$$
V_{1}-V_{2}=\frac{k}{8 \pi e}\left(\frac{E_{1}^{2}}{n_{1}}+\frac{E_{2}^{2}}{n_{2}}\right),
$$

wobei für die Oberflächenladungen in KG-Zuständen gilt :

$$
q=\frac{k}{4 \pi}\left(E_{1}-E_{2}\right) .
$$

(Wir folgen hier eng den Gedankengängen von $\mathrm{T}$ a y lor und Mitarb., da diese Grundlagen für die folgenden Ausführungen unentbehrlich sind ${ }^{8}$.)

Im Gleichgewichtsfall (Index 0) kann man nun die elektrostatischen Potentiale zu beiden Seiten gleichsetzen und erhält

$$
\begin{gathered}
\Phi_{1}=\Phi_{2}=\Phi ; \quad V_{10}=V_{20}=\Phi / e ; \\
E_{10}=-E_{20}=-2 \pi q_{0} / k .
\end{gathered}
$$

Nach (7) und (8) gilt bei einer angelegten äußeren Spannung $V_{\text {a }}$

$$
E_{1}=-2 \pi q / k-V_{\mathrm{a}} e n / q ; \quad E_{2}=2 \pi q / k-V_{\mathrm{a}} e n / q .
$$

Wird nun $E_{1}=E_{2}=0$, so erreicht $V_{\text {a }}$ den kritischen Durchbruchswert

$$
V_{\mathrm{c}}=\frac{2 \pi}{k e n} q_{0}^{2} .
$$

Mit Gl. (10) ergibt das

$$
V_{\mathrm{c}}=\frac{k}{2 \pi e n} E_{10}^{2} .
$$

Nun ist nach (6) für den Gleichgewichtsfall

$$
E_{10}^{2}=E_{20}^{2}=8 \pi \text { e } n V_{10} / k \text {. }
$$

Das ergibt mit (13a)

$$
V_{\mathrm{c}}=4 V_{10} \quad \text { resp. mit (9) } V_{\mathrm{c}}=4 \Phi / e .
$$

In anderen Worten, die Sperrspannung an einer KG könnte so nur sehr klein sein, da $\Phi$ normalerweise nur Bruchteile eines Elektronenvolts betragen kann. Die KG-Terme liegen, wie wir gesehen haben, etwa in der Mitte des verbotenen Bandes.

Da aber in Wirklichkeit hohe Sperrspannungen auftreten, können wir die eingeführte Voraussetzung, $q_{0}=$ const, oder die Annahme, daß die Anzahl der gebundenen Ladungsträger unabhängig von der Feldstärke ist, nicht aufrechterhalten.

T a y lor und Mitarb. ${ }^{8}$ haben als erste Schottkys Sperrschicht-Theorie ${ }^{29}$ auf die KG-Zone angewandt.

Es läßt sich so zeigen, daß mit der Querspannung die Zahl der in KG-Niveaus gebundenen Elektronen anwächst, während die Zahl der Defektelektronen abnimmt.

Die Stromgleichung für die Doppelschicht ist gemäß Schottky :

$$
\begin{aligned}
i=e \mu E\left[n_{\mathrm{B}}-n_{\mathrm{A}} \exp \left(\mp e V_{\mathrm{AB}} / k T\right)\right] \\
\cdot\left[1-\exp \left(\mp e V_{\mathrm{AB}} / k T\right)\right]^{-1}
\end{aligned}
$$

( $n_{\mathrm{A}}$ Störstellendichte am untern Rande der KG-Sperrschicht, $n_{\mathrm{B}}$ Störstellendichte im oberen Teil der KG-Sperrschicht, $e$ Elementarladung, $\mu$ Ladungsträgerbeweglichkeit, $E$ Feldstärke in der KG-Sperrschicht, $V_{\mathrm{AB}}$ Spannungsabfall an der $\mathrm{KG}, k t$ Wärmeenergie).

29 W. S c hot t k y, Z. Phys. 118, 546 [1941/42]. 
Das Minuszeichen bezieht sich auf Elektronen-, das Pluszeichen auf Defektelektronenleitung.

Da die Elektronenkonzentration am Fuße der KG (Index A) gleich der Gleichgewichtskonzentration $n_{\mathrm{e} 0}$ ist, und da ferner für KG-Sperrschichten

$$
e V_{\mathrm{AB}} \gg k T
$$

gilt, so wird

$$
i_{\mathrm{e}}=e \mu_{\mathrm{e}} E\left[n_{\mathrm{eB}}-n_{\mathrm{e} 0} \exp \left(-e V_{\mathrm{AB}} / k T\right)\right] .
$$

Dies ist eine Stromgleichung, ähnlich derjenigen für eine P-N-Verbindung. Führt man hier nun die Felder $E_{1}$ und $E_{2}$ auf beiden Seiten der KG-Doppelschicht ein und ferner $V_{\mathrm{a}}=V_{1}-V_{2}$ (siehe Abb. 11), so erhält man für Elektronen- und Defektelektronenstrom nach Elimination von $n_{\mathrm{eB}}$ :

$$
\begin{gathered}
i_{\mathrm{e}}=-\mu_{\mathrm{e}} e \frac{E_{1} E_{2}}{E_{1}-E_{2}} n_{\mathrm{e} 0} \exp \left(-e V_{2} / k T\right) \\
\cdot\left[1-\exp \left(-e V_{\mathrm{a}} / k T\right)\right], \\
i_{\mathrm{h}}=e \mu_{\mathrm{h}} E_{1} E_{2} \frac{n_{\mathrm{h} 2} \exp \left(-e V_{1} / k T\right)-n_{\mathrm{h} 1} \exp \left(-e V_{2} / k T\right)}{E_{1} \exp \left(-e V_{1} / k T\right)-E_{2} \exp \left(-e V_{2} / k T\right)} .
\end{gathered}
$$

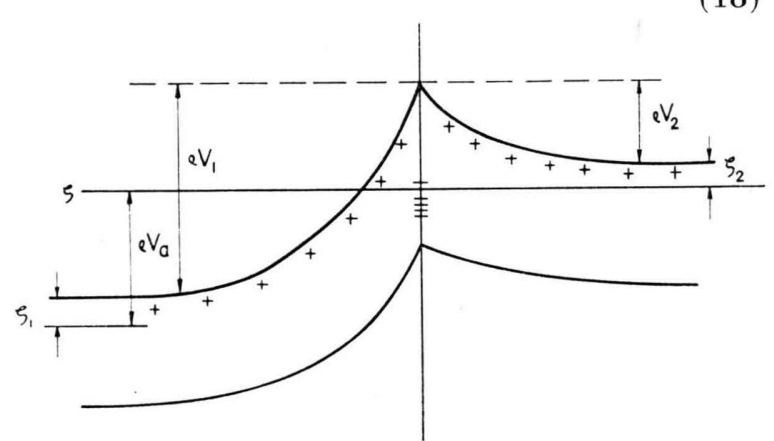

Abb. 11. Bändermodell einer Korngrenze mit transversaler Polarisation. e $V_{\mathrm{a}}=e V_{1}-e V_{2}$.

Die Beziehung (17) für den Elektronenstrom zeigt, daß mit wachsender äußerer Spannung $V_{\text {a }}$ der Elektronenstrom nur von $V_{2}$ abhängig ist. - Mit $V_{2}$ anwachsend muß $i_{\mathrm{h}}$ abnehmen, da sich dann der KGSprung erhöht. - Nun ist aber in Wirklichkeit das KG-Potential auf einer Seite, $\mathrm{eV}_{2}$, nicht nur von $q$, der Anzahl der Elektronen in KG-Niveaus, abhängig. Da $q$ von $e V_{\text {a }}$ abhängig ist, sind auch $e V_{1}$ und $e V_{2}$ von $e V_{\text {a }}$ abhängig. Wie schon $\mathrm{F}$ a n bemerkte, wirkt die Erhöhung der negativen Ladung in der KG-Zone im Sinne einer Verminderung der Abnahme von $V_{2}$.

Aus Gl. (12) ergibt sich für $V_{2}=V_{20}=\Phi / e$ oder $E_{2}=E_{20}$ unter Berücksichtigung von (9)

$$
2 \pi q / k-e n V_{\mathrm{a}} / q=2 \pi q_{0} / k
$$

oder

$$
q=q_{0} / 2 \cdot\left[1+\left(1+e V_{\mathrm{a}} / \Phi\right)^{1 / 2}\right],
$$

da k e $n / 2 \pi=q_{0}^{2} / V_{\mathrm{c}}=q_{0}^{2} / 4 \Phi$ für den Fall $n_{1}=$ $n_{2}=n$ und im Gleichgewicht $V_{10}=V_{20}=\Phi / e$.

Die Funktion $q=f\left(V_{\mathrm{a}}\right),(20)$, zeigt die Abhängigkeit der Zahl der gebundenen Ladungsträger in der $\mathrm{KG}$ von der angelegten Spannung an die KG. $q$ nimmt danach mit $V_{\mathrm{a}}=V_{1}-V_{2}$ zu.

Das heißt aber, daß für $q \neq q_{0}$ auch $V_{1}$ und $V_{2}$ mit $V_{\text {a }}$ dem Absolutbetrag nach zunehmen.

Setzen wir (12) in (6) ein, so ist nämlich

$$
\begin{aligned}
& V_{1}=(k / 8 \pi e n)\left(_{E_{1}{ }^{2}}^{\left(2 \pi q / k+e n V_{\mathrm{a}} / q\right.}\right)^{2}, \\
& V_{2}=(k / 8 \pi e n) \underbrace{(\underbrace{2 \pi q / k-e n V_{\mathrm{a}} / q})^{2} .}_{E_{2}{ }^{2}}
\end{aligned}
$$

Die Form der Funktionen $V_{1}=f\left(V_{\mathrm{a}}\right), V_{2}=f\left(V_{\mathrm{a}}\right)$ einschließlich der Änderung über $q$ geht durch Einsetzen von (20) in (21) und (22) hervor:

$$
\begin{gathered}
V_{1}=(k / 8 \pi e n)\left\{\frac{\pi q_{0}}{k}\left[1+\left(1+e V_{\mathrm{a}} / \Phi\right)^{1 / 2}\right]\right. \\
\left.+2 e n V_{\mathrm{a}} /\left[1+\left(1+e V_{\mathrm{a}} / \Phi\right)^{1 / 2}\right] q_{0}\right\}^{2}, \\
V_{2}=(k / 8 \pi e n)\left\{\frac{\pi q_{0}}{k}\left[1+\left(1+e V_{\mathrm{a}} / \Phi\right)^{1 / 2}\right]\right. \\
\left.\quad-2 e n V_{\mathrm{a}} /\left[1+\left(1+e V_{\mathrm{a}} / \Phi\right)^{1 / 2}\right] q_{0}\right\}^{2} \quad(24) \\
\text { oder } V_{1 / 2}=\frac{\pi q_{0}^{2}}{8 e n k} \overbrace{\left.2\left[1+\left(1+e V_{\mathrm{a}} / \Phi\right)^{1 / 2}\right]+e V_{\mathrm{a}} / \Phi\right\}}\} \\
\pm \frac{V_{\mathrm{a}}}{2}+\frac{e n k}{2 \pi q_{0}^{2}} V_{\mathrm{a}}^{2} \underbrace{2\left[1+\left(1+e V_{\mathrm{a}} / \Phi\right)^{1 / 2}\right]+e V_{\mathrm{a}} / \Phi}, \\
V_{1 / 2}=\frac{\pi q_{0}^{2}}{8 e n k} a \pm \frac{V_{\mathrm{a}}}{2}+\frac{e n k}{2 \pi q_{0}^{2}} V_{\mathrm{a}}^{2} \frac{1}{a} .
\end{gathered}
$$

Das Minuszeichen bezieht sich hier auf $V_{2}$.

Demnach sind $V_{1}$ und $V_{2}$ Funktionen von $V_{\mathrm{a}}^{1 / 2}$, $V_{\mathrm{a}}$ und, wenn $e V_{\mathrm{a}} / \Phi<1$, von $V_{\mathrm{a}}^{2}$.

Der Unterschied in den Beträgen zwischen $V_{1}$ und $V_{2}$ ist, wie anzunehmen, linear in $V_{\mathrm{a}} . \quad V_{1}$ und $V_{2}$ wachsen aber quadratisch mit $V_{\mathrm{a}}$, wenn die $q$ Variation berücksichtigt wird und die Vorspannung noch gering ist. Hierdurch wird die starke Bedeutung der Ladungsanhäufung durch Polarisation an der KG deutlich.

Wir wollen nun die Elektronenkonzentration $n_{\mathrm{eB}}$ auf der Höhe der KG-Sperrschicht abschätzen:

Setzen wir gemäß Gl. (15) eine Strom-Spannungsbeziehung für beide KG-Seiten an, so ergibt sich nach einiger Algebra (vgl. ${ }^{7}$ )

$$
n_{\mathrm{eB}} \cong-n_{\mathrm{e} 0} \frac{E_{2}}{E_{1}-E_{2}} \exp \left(-e V_{2} / k T\right),
$$

wenn

$$
V_{\mathrm{a}} \gg k T / e \text {. }
$$


Die Defektelektronenkonzentration wird

$$
n_{\mathrm{hB}} \cong 2 n_{\mathrm{h} 0} \exp \left(e V_{2} / k T\right) .
$$

$E_{1}-E_{2}=-q$ ist die Zahl der Elektronen in KGNiveaus. Setzen wir zur Abschätzung des qualitativen Verhaltens bei Vorspannungsänderung einfach $q=q_{0}=$ const, so ergibt die Differentiation von $n_{\mathrm{eB}}$ und $n_{\mathrm{hB}}$ nach $V_{\mathrm{a}}$ :

$$
\begin{gathered}
\frac{\partial n_{\mathrm{eB}}}{\partial V_{\mathrm{a}}} \cong V_{2}^{1 / 2} \frac{n_{\mathrm{e} 0}}{q} \frac{e}{k T}\left(\frac{k T}{2 e V_{2}}-1\right) \exp \left(-e V_{2} / k T\right) \frac{\partial V_{2}}{\partial V_{\mathrm{a}}}, \\
\frac{\partial n_{\mathrm{hB}}}{\partial V_{\mathrm{a}}} \cong 2 n_{\mathrm{h} 0} \frac{e}{k T} \exp \left(e V_{2} / k T\right) \frac{\partial V_{2}}{\partial V_{\mathrm{a}}} .
\end{gathered}
$$

Für $V_{2} \gg k T / e$ ist (29) positiv, während (30) negativ wird, was bedeutet, daß $n_{\mathrm{eB}}$ mit $V_{\mathrm{a}}$ anwächst, während $n_{\mathrm{hB}}$ ceteris paribus abnimmt. Wie bereits gesagt, ist die Voraussetzung $q=$ const nicht erfüllt. Berücksichtigt man die Erhöhung der Zahl der KGNiveaus mit wachsender Spannung $V_{\mathrm{a}}$ durch Einführen von $q\left(V_{\mathrm{a}}\right)$ nach (20) in $n_{\mathrm{eB}}$ nach Gl. (27), so ist

$$
n_{\mathrm{eB}} \cong \frac{n_{\mathrm{e} 0}}{q_{0}} \frac{2 V_{2}^{1 / 2}}{\left[1+\left(1+e V_{\mathrm{a}} / \Phi\right)^{1 / 2}\right]} \exp \left(-e V_{2} / k T\right),
$$

und die Differentiation ergibt

$$
\begin{array}{r}
\frac{\partial n_{\mathrm{eB}}}{\partial V_{\mathrm{a}}} \cong \frac{n_{\mathrm{e}_{0}}}{q_{0}} V_{2}^{1 / 2} \frac{2 e}{k T\left[1+\left(1+e V_{\mathrm{a}} / \Phi\right)^{1 / 2}\right]}\left\{\left(\frac{k T}{2 e V_{2}}-1\right) \frac{\partial V_{2}}{\partial V_{\mathrm{a}}}\right. \\
\left.-\frac{k T}{2 \Phi}\left(1+e V_{\mathrm{a}} / \Phi\right)^{-1 / 2}\left[1+\left(1+e V_{\mathrm{a}} / \Phi\right)^{1 / 2}\right]^{-1}\right\} \\
\cdot \exp \left(-e V_{2} / k T\right) .
\end{array}
$$

Wie vorhin bleibt der Anstieg von $n_{\mathrm{eB}}$ mit $V_{\mathrm{a}}$ erhalten, da $\partial n_{\mathrm{eB}} / \partial V_{\mathrm{a}}$ nur ein weiteres positives Glied erhält.

Die angelagerten Elektronen bewirken also eine weitere Erhöhung des KG-Potentials. Die angelegte Spannung wird nach Erreichen eines Sättigungswertes jedoch zum Zener-Durchbruch durch beide Sperrschichten führen.

Zusammenfassend ist zu sagen, daß die KG-Dreifachschicht (zwei Doppelschichten mit gemeinsamer Mittelschicht) im allgemeinen den Charakter einer $\mathrm{N}-\mathrm{P}-\mathrm{N}$-Schichtung aufweist. Hier wie dort wird der Ladungsträgerstrom von Material getragen, das eine größere Leitfähigkeit für einen Typ dieser Ladungsträger (hier Elektronen) außerhalb dieser Zone hat als innerhalb. In der KG-Zone herrscht also ein starker Trägerdichtengradient.

Wie im Falle einer $\mathrm{P}-\mathrm{N}$-Verbindung ist hier auch der integrierte spezifische Widerstand größer als im umgebenden Kristall. Anders jedoch, als im Fall der P-N-Schichtung, besteht hier eine Span- nungsabhängigkeit der Anlagerungsquote für freie Ladungsträger (Elektronen) infolge der verstärkten „Fallenwirkung“ in KG-Niveaus.

\section{Beeinflussung des Korngrenzenfeldes durch Trägerinjektion und Licht}

Die vorangehenden Betrachtungen führen zu der Annahme, daß die KG-Zone als Ort starker Ladungsträgeranhäufung, die durch eine Potentialschwelle ausgezeichnet ist, für sich elektronisch beeinflußbar ist und daß eine solche Zone in besonderer Weise polarisierbar ist. Damit stellt sie eine Elektrode innerhalb des Kristalls dar, die durch Potentialverschiebung oder als Ort für Trägerinjektion die Rolle einer Steuerelektrode übernehmen kann.

Wir erörtern hier verschiedene Methoden:

a) Anordnung zur Modulation des KG-Querstromes nach Art eines Fadentransistors mit auf die KG-Zone aufgesetzter Spitze (Abb. 12). Auf einer Seite des Querfeldes injizierte Defektelektronen können z. B. durch gleichzeitige Injektion von Defektelektronen in die KG-Zone zur Drift zur Gegenelektrode veranlaßt werden. Dadurch tritt Senkung des KG-Feldes und Erhöhung des Querstromes ein. Ebenso kann bei negativem Potential der KG-Zone die „Fallenrate“ für Elektronen erniedrigt werden und eine vom Querfeld unabhängige Stromdichte eingestellt werden (Sättigungswert).

b) Modulation durch Lichteinfall in die KG-Zone. Ähnliche Effekte können beobachtet werden durch Paarerzeugung in der KG-Zone, verursacht durch Lichteinfall im richtigen Frequenzbereich. Die erzeugten Elektronen können zunächst an KG-Niveaus gebunden werden und tragen dann zur Erhöhung des Potentialsprungs bei. Anderseits können sie zur Kollektorseite diffundieren.

Lichteinwirkung auf die KG-Zone ist von stärkerer Einwirkung auf den Querstrom, wenn die Polarisation der KG so stark ist, daß dadurch eine Störung des Gleichgewichts und damit Erhöhung des Querstromes gegeben sind. Lichteinfall in die KG-Zone ergibt bezügl. Trägerbewegung einen höheren Wirkungsgrad als Lichteinfall auf eine monokristalline Struktur. Dies infolge der höheren Einfangquote für Ladungsträger in KG-Niveaus. Ähnlich liegen die Verhältnisse bei Beleuchtung von $\mathrm{P}-\mathrm{N}$-Inversionsschichten.

Daher können Photodioden und -Transistoren, bei denen entsprechend orientierte und polarisierte KG 
im Strahlengang liegen, einen hohen Wirkungsgrad aufweisen. In anderen Worten, Befreiung einzelner Träger aus höheren KG-Niveaus führt zu einer Modulation der Lage der durch Vorspannung erzeugten Quasi-Fermi-Niveaus.

Wir können hier nicht näher auf die Wirkungsweise dieser KG-Modulation eingehen, was einer späteren Arbeit vorbehalten bleiben soll.

c) Steuerung eines Stromes durch einen Bikristall, in dem eine KG-Ebene quer zum Stromweg liegt. Eine KG kann leicht so hergestellt werden, daß sie an der Bikristall-Oberfläche als gerade Linie auftritt. Die KG-Ebene kann dabei senkrecht zur Schnittfläche angeordnet sein. Auf diese Weise ist die Zone höherer Energie, welche die KG-Ebene darstellt, auf eine schmale Schicht mitten im sonst perfekten Kristall konzentriert. Bei Querpolarisation dieser Ebene ist, wie wir gesehen haben, beiderseits eine Inversionsschicht und eine Zone positiver Raumladung aufgebaut. Dazu herrscht in Richtung der KG-Ebene höhere Leitfähigkeit (Abb. 3), so daß die KG-Ebene auf ein definiertes Potential gegenüber dem übrigen Kristallkörper gebracht werden kann*.

\section{Querstrom-Modulation}

Ergebnisse von Messungen mit 3 Elektroden an KG-Kristallen bestätigen die Vermutungen.

Abb. 12 stellt eine 3-Sonden-Anordnung dar. Der Kristall ist hier aus einem polykristallinen Block herausgeschnitten (Silicium).

Es wurde ein Mikromanipulator mit speziell gebauten Manipulatorarmen verwandt. Die Einstellung kann hier so erfolgen, daß die 3-Sonden-Anordnung über Manipuliergetriebe unter bestimmtem Druck auf den Kristall aufgesetzt wird und nach erfolgter Messung der Kristall mittels seines Tisches gesenkt wird. Sodann wird der Tisch um eine bestimmte Länge unter dem Mikroskop verschoben, um die

* Forschungen von anderer Seite ${ }^{30}$ haben diesen Befund inzwischen bestätigt. Germanium-Bikristalle, die aus (100)Saatkristallen hergestellt wurden, zeigten die oben genannten Eigenschaften. Die Verdrehungswinkel betrugen zwischen 5 und 15 Grad. Die beiden Saatkristalle waren an einem Molybdänkeil angeordnet. Die Keilebene lag in der gemeinsamen (110)-Richtung der Kristalle. Messungen parallel zur KGEbene ergaben Werte zwischen $2 \cdot 10^{3}$ und $10^{5} \mathrm{Ohm}$ integralen Widerstand. Die gleiche Kontaktfläche ergab über die gleiche Kristallänge am perfekten Kristall $10^{6} \mathrm{Ohm}$.

30 A. G. T w e e t, Phys. Rev. Bull. 29, 17 [1954].
Sonden auf einer anderen Kristallstelle, aber unter Konstanthaltung der Geometrie und des Druckes, aufsitzen zu lassen. Anschließend wird der Manipulatortisch in die alte Höhe verschoben, wobei die Nadelstellung wieder sehr genau der vorigen entspricht, und zwar mit gleichem Kontaktdruck, da die Manipulierarme Federn enthalten und die vertikale Tischverschiebung mittels einer Mikrometeruhr gemessen wird.

Abb. 13 zeigt das Resultat einer solchen Messung. Aufgetragen ist als Ordinate der Querstrom durch die KG, als Abszisse die Querspannung. Parameter ist der Strom in die auf der KG aufsitzende Elektrode. Es handelt sich hier um einen GermaniumBikristall, dessen perfekte Seiten je einen spezifischen Widerstand von $10 \mathrm{Ohm} \cdot \mathrm{cm}$ haben. Der Leitungscharakter ist N-Typ. Es sind zwei Meßfelder aufgetragen: a) schraffiert: außerhalb der KG-Zone, b) innerhalb der KG-Zone. Man sieht, daß auch außerhalb der KG-Zone die 3. Spitzenelektrode durch normale Minoritätsträger-Injektion eine Stromänderung im Außenkreis herbeiführt $\left(\alpha_{i}=\Delta I_{1} / \Delta I_{2}=1\right)$. Im Falle der Injektion in die KG-Ebene tritt jedoch Erhöhung des $\alpha_{i}$-Wertes auf und zugleich ist die Impedanz an den Elektroden quer zur KG erhöht.

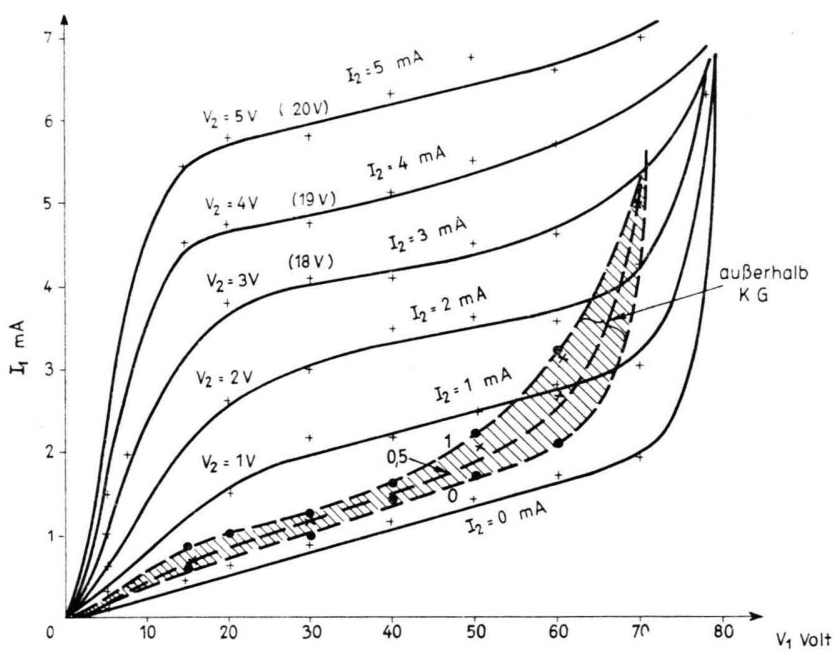

Abb. 13. Kennlinienfeld eines KG-Transistors. - Schraffierte Zone: außerhalb der KG-Linie. Germanium, $5 \mathrm{Ohm} \cdot \mathrm{cm}$, N-Typ. Parameter ist Strom in die KG-Zone. Eingetragen einige $V_{2}$-Werte. $V_{2}$ entsprechend $V_{1}$ für $V_{1} \geqq 40 \mathrm{~V}$.

Abb. 14 stellt das gleiche für einen Silicium-Bikristall dar. Der Unterschied zwischen den $\alpha_{i}$-Werten außerhalb und innerhalb der KG-Zone ist hier beträchtlich. Einige $V_{2}$-Werte sind ebenfalls angegeben. 
Die Sättigungszone ist ausgeprägter und erstreckt sich von 20 bis 100 Volt Kollektorspannung. Die Spannungswerte $V_{2}$ am Korngrenzenkreis (gemessen in bezug auf eine Seite des polarisierten Bikristalls) nehmen mit wachsender Spannung $V_{2}$ (quer zur KG) ab.

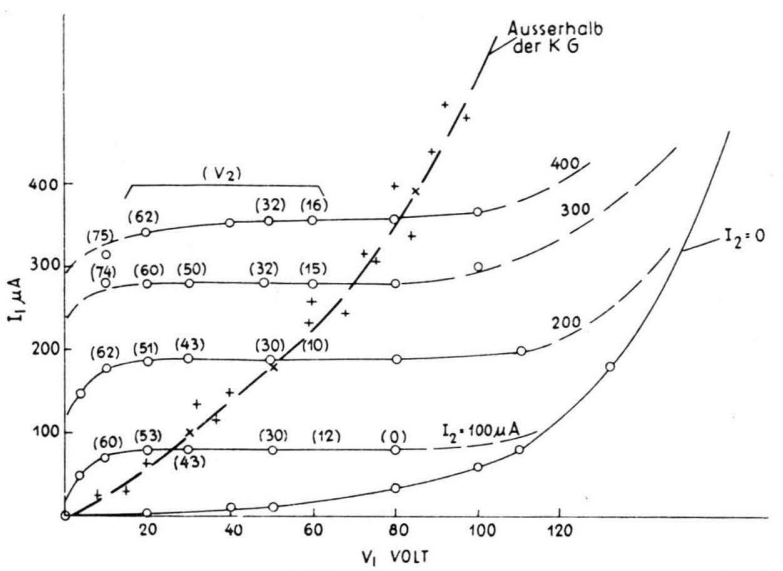

Abb. 14. Kennlinienfeld eines Silicium-KG-Transistors. Außerhalb der KG-Zone (d. h. mittlere Sonde nicht auf KG aufsitzend) keine Aufspaltung in Kennlinienbündel. Bei Injektion in KG tritt Sättigung des Querstromes auf. Silicium, $5 \mathrm{Ohm} \cdot \mathrm{cm}$, N-Typ.

Es ist typisch für das KG-Verhalten, daß Stromverstärkungswerte $\alpha$ größer 1 auch in einer Anordnung gemessen werden können, bei der die KG auf Bezugspotential gelegt ist.

Abb. 15 zeigt diesen Fall. Ein solches Verhalten ist nicht im Falle einer $\mathrm{N}-\mathrm{P}-\mathrm{N}$-Verbindung, die auf Störstellendosierung beruht, herzustellen. Der hier verwandte Bikristall ist der oben beschriebene, bei $\operatorname{dem} \Theta_{1}=5^{\circ}$ beträgt. Selbstverständlich ergibt eine Dreisondenanordnung mit Emitter-Bezugspunkt die z. B. in Abb. 16 angegebene Kennlinie mit wesentlich höheren $\alpha$-Werten.

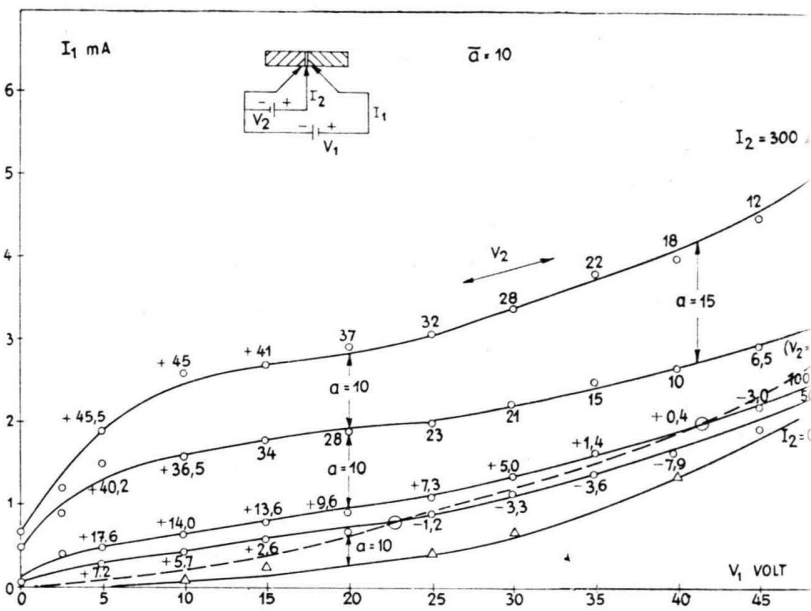

Abb. 16. Gleiches Material wie in Abb. 15, jedoch Messung in 3-Sondenanordnung und Emitter-Basis.

Abb. 17 stellt eine schematische Anordnung und das entsprechende Bändermodell eines solchen KGTransistors dar.

Die starke Aufkrümmung des Leitungs- und Valenzbandes in der KG-Zone erzeugt eine typische „Haken“-Konfiguration (Shockley) mit entsprechender Ladungsträger-Dichte-Verschiebung. (Höhere Anzahl befreiter Elektronen pro injiziertes Defektelek-

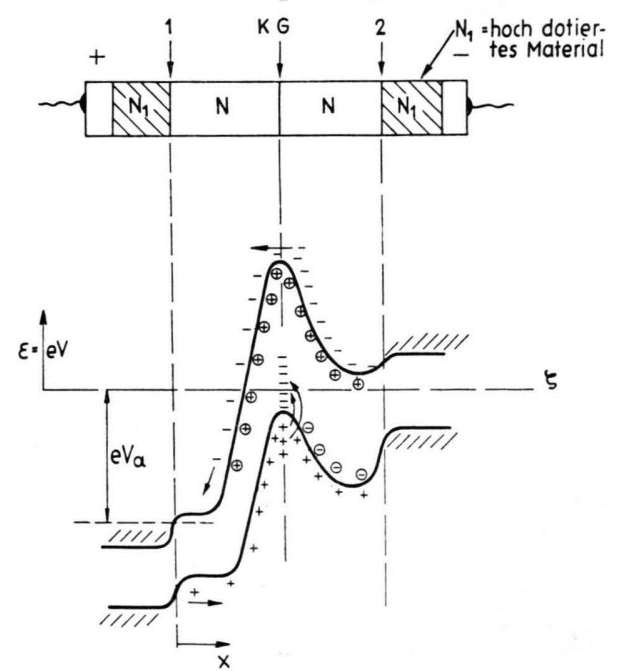

Abb. 17. Anordnung und Bändermodell einer Korngrenze mit angelegter Querspannung $V_{\mathrm{a}}$. Veranschaulichung des „Haken“. Effekts nach Shockley. 
tron.) Die Ausnutzung solcher Zonen ist auch in $\mathrm{N}-\mathrm{P}-\mathrm{N}$-Strukturen möglich, um die Stromverstärkungswirkung eines Kollektors in geerdeter Basisschaltung auszunutzen (Abb. 18).

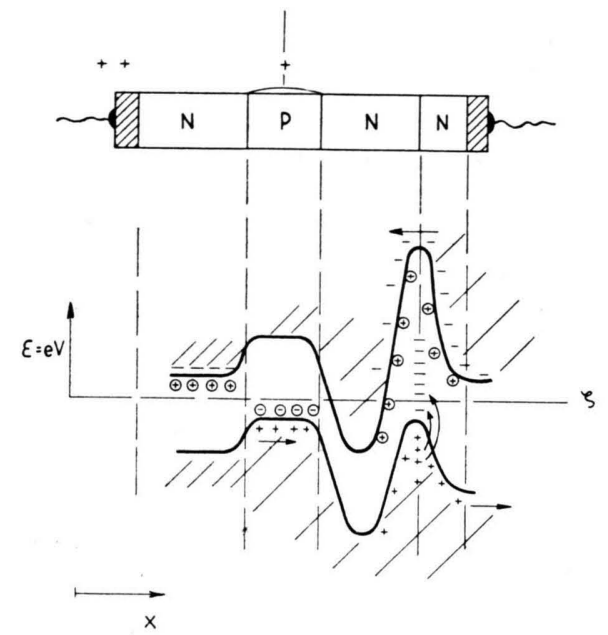

Abb. 18. Schematische Darstellung und Bändermodell für einen $\mathrm{N}-\mathrm{P}-\mathrm{N}$-Transistor mit KG-Zone vor Kollektor.

Wie im Falle der Polarisation einer $\mathrm{N}-\mathrm{P}-\mathrm{N}$ Struktur kommt es darauf an, die durch die erste Sperrschicht in die P-Typ-Zone injizierten Minoritätsladungsträger (Flußgebiet) durch Steuerung der Mittelschicht, die gegen die zweite Zone (N-Typ) auf hohem Potential liegt, zu beeinflussen.

Bei steigendem Kollektorpotential kann das hier, wie z. B. in Abb. 15 zu sehen, zu einem Polaritätswechsel der Emitterschicht führen $\left(V_{\mathrm{e}}=0\right.$-Linie). Inwieweit bei Auffüllung aller KG-Niveaus mit Elektronen wieder Injektion von Defektelektronen möglich ist, hängt von den komplizierten Potentialverhältnissen innerhalb der Schichten ab.

Wie wir oben gesehen haben, können hohe Stromverstärkungswerte auf der Basis der erhöhten Fallenrate und daraus resultierender, hoher Trägerdichte in der KG-Zone verstanden werden.

Wird die KG-Zone polarisiert, so erfahren die Größen

$n_{\mathrm{eB}}$, Elektronendichte in der $\mathrm{KG}$,

$V_{\mathrm{AB}}$, Spannungsabfall an der $\mathrm{KG}$

in der Stromgleichung (16)

$$
i_{\mathrm{e}}=e \mu_{\mathrm{e}} E\left[n_{\mathrm{eB}}-n_{\mathrm{e} 0} \exp \left(-e V_{\mathrm{AB}} / k T\right)\right]
$$

starke Änderungen. Die KG-Sperrzone wird im wesentlichen durch $V_{2}$ bestimmt (Abb. 11). Eine Abschätzung der Leitfähigkeitsänderung durch $V_{2}$-Variation kann wie folgt durchgeführt werden:
Mit den Beziehungen

$$
E_{1}-E_{2}=-q, E_{2} \cong V_{2}^{1 / 2}
$$

wird Gl. (27) zu

$$
n_{\mathrm{eB}} \simeq n_{\mathrm{e} 0} \frac{V_{2}^{1 / t}}{q} \exp \left(-e V_{2} / k T\right) .
$$

Daher ist

$$
\frac{\partial n_{\mathrm{eB}}}{\partial V_{2}} \cong{ }^{\prime} n_{\mathrm{e} 0} \alpha V_{2}^{1 / 2}\left[\frac{1}{2 \alpha V_{2}}-1\right] \exp \left(-\alpha V_{2}\right),
$$

wo $\alpha=e / k T$.

Da $V_{2} \gg 1 / \alpha$, erhält man

$$
\partial n_{\mathrm{eB}} / \partial V_{2} \simeq-\frac{n_{\mathrm{e} 0}}{q} \alpha V_{2}^{1 / 2} \exp \left(-\alpha V_{2}\right),
$$

ebenso für die Defektelektronendichte

$$
\partial n_{\mathrm{hB}} / \partial V_{2} \cong 2 \alpha n_{\mathrm{h} 0} \exp \left(\alpha V_{2}\right) \text {. }
$$

Nun können wir diese Trägerdichteschwankungen mit $V_{2}$ in die modifizierte Gleichung für die Leitfähigkeitsänderung infolge Fallenwirkung eintragen : $\Delta \sigma=e \mu_{\mathrm{e}}\left(\Delta n_{\mathrm{e}}+\Delta n_{\mathrm{et}}\right)+e \mu_{\mathrm{h}}\left(-\Delta n_{\mathrm{h}}+\Delta n_{\mathrm{ht}}\right)$,

$\Delta n_{\text {et }}$ Änderung der Elektronendichte in Fallen (Traps),

- $\Delta n_{\mathrm{ht}}$ Änderung der Defektelektronendichte (abnehmend mit wachsendem Querfeld).

Da für diese durch die Querspannung bestimmten Änderungen $V_{2}$ eine Funktion von $V_{\mathrm{a}}$ ist, entsteht die Bruttogleichung:

$$
\begin{aligned}
\Delta \sigma & \cong e \mu_{\mathrm{e}}\left[\Delta n_{\mathrm{e}}-{ }^{n_{\mathrm{e} 0}} \alpha \mid V_{\mathrm{a}}{ }^{1 / 2} \exp \left(-\alpha\left|V_{\mathrm{a}}\right|\right)\right] \\
& +e \mu_{\mathrm{h}}\left[-\Delta n_{\mathrm{h}}+2 n_{\mathrm{h} 0} \alpha \exp \left(\alpha\left|V_{\mathrm{a}}\right|\right)\right] .
\end{aligned}
$$

Die Leitfähigkeit der KG-Zone nimmt also nur wenig zu durch eine Änderung der Elektronendichte in Fallen, dagegen hat eine Änderung der Defektelektronendichte starken Einfluß.

Wir können nun gemäß Gl. (38) die Leitfähigkeitsschwankung in der KG-Zone durch eine StromSpannungsbeziehung ausdrücken.

$\Delta I_{2}$ und $\mathrm{AV}_{2}$ seien die Bezeichnungen für Stromund Spannungsänderung für die KG-Zone selbst, durch getrennte Polarisation in bezug auf eine Seite des Querfeldes:

$$
\begin{aligned}
& \Delta I_{2} \cong\left\{e \mu_{\mathrm{e}} \Delta n_{\mathrm{e}}\left[1-\frac{n_{\mathrm{e}_{0}}}{\Delta n_{\mathrm{e}}} \frac{\alpha}{q}\left|V_{\mathrm{a}}\right|^{1 / 2} \exp \left(-\alpha\left|V_{\mathrm{a}}\right|\right)\right]\right. \\
& \left.+e \mu_{\mathrm{h}} \Delta n_{\mathrm{h}}\left[-1+\frac{2 n_{\mathrm{h}_{0}}}{\Delta n_{\mathrm{h}}} \alpha \exp \left(\alpha\left|V_{\mathrm{a}}\right|\right)\right]\right\}\left(V_{2}+\Delta V_{2}\right) .
\end{aligned}
$$

Dies ist aber eine Gleichung, welche derjenigen für zwei in Serie geschaltete $\mathrm{P}-\mathrm{N}$-Gleichrichter ent- 
spricht. $\Delta V_{2}+V_{2}$ als zusätzlicher Parameter ergibt hier die Aufspaltung in ein Bündel von Charakteristiken:

$$
\begin{aligned}
\Delta I \cong & \left\{\text { const }\left[1-\text { const }\left|V_{\mathrm{a}}\right|^{1 / 2} \exp \left(-\alpha\left|V_{\mathrm{a}}\right|\right)\right]\right. \\
& \left.+ \text { const }\left[-1+\text { const } \exp \left(\alpha\left|V_{\mathrm{a}}\right|\right)\right]\right\}\left(V_{2}+\Delta V_{2}\right) .
\end{aligned}
$$

Es hat den Anschein, daß Elektronen in KGNiveaus in einem semistabilen Zustand verharren können, wie oben beschrieben ${ }^{31}$. Umladungsprozesse, an denen diese Ladungsträger teilnehmen, können erheblich kürzere Zeitkonstanten aufweisen als normale Trägerinjektion und -Befreiung. Während einerseits der schädliche Einfluß der Fallen im KGFeld auf Beweglichkeit und Lebensdauer der Träger bekannt ist, ist anderseits ein gutes Frequenzverhalten bei Umladungsprozessen zu erwarten, in denen Träger in semistabilen Zuständen beteiligt sind.

Untersuchungen über den Einfluß der KG-Zonen auf Beweglichkeit, Lebensdauer, Rekombination usf. haben die Orientierung der KG-Zone und den Ladungstransport relativ zur KG-Ebene im Kristall zu berücksichtigen.
Die genauere Theorie der KG-Modulation, sei es durch einfache Polarisation der Mittelschicht (Ohmsche Kontakte), sei es durch zusätzliche Ladungsträgerinjektion, ist noch im Sinne einer Kennliniendarstellung auszuarbeiten, die derjenigen für den $\mathrm{N}-\mathrm{P}-\mathrm{N}$-Transistor entspricht. Es sollte daraus die Anzahl der notwendigen Akzeptorterme (KG-Niveaus) und damit die Orientierung $\left(\Theta_{1} ; \Theta_{2} ; \Theta_{3}\right)$ gefolgert werden können.

Für freundliche Hilfe bei der Durchführung der ersten Arbeiten auf diesem verzweigten Gebiet habe ich den Kollegen der Signal Corps Laboratorien, Fort Monmouth, zu danken. Dr. P. H. K e c k und Dr. H. K e d e s d y (Chemical Physics Branch) stellten Geräte und Hilfskräfte für die Herstellung von Si-Bikristallen, für elektrische Messungen mit Mikromanipulatoren und Röntgenapparate für die Laue-Aufnahmen zur Orientierungsmessung zur Verfügung. Mr. H. O wens unterstützte meine Arbeiten durch Aufbau einer GermaniumBikristall-Ziehapparatur in der Solid State Branch. R. S e i densticker danke ich für ausgezeichnete Mitarbeit bei der Herstellung der Bikristalle und A. M c D on a ld für Mitarbeit bei mühsamen Mikrosondenmessungen. Ihnen allen sowie Dr. W. K a i s e r und Dr. G. W olf f danke ich für Diskussionen. Prof. H. Y. F a n danke ich für briefliche Auskunft betreffend seine Arbeit ${ }^{7}$.

${ }^{31}$ W. Shockley u. W. T. R e a d, Phys. Rev. 87, No. 5, [1952].

\section{NOTIZEN}

\section{Die Newtonsche Abbildungsgleichung des magnetischen Sektorfeldes bei Berücksichtigung des Streufeldes}

Von Lothar A. König*

Max-Planck-Institut für Chemie, Mainz

(Z. Naturforschg. 10a, 652-654 [1955]; eingegangen am 12. Juli 1955)

Die Abbildungseigenschaften magnetischer Sektorfelder bei Vernachlässigung des Streufeldes sind seit langem bekannt. Nachdem C l a s s e ${ }^{1}$ und S t e p h en s ${ }^{2}$ die Fokussierungseigenschaften für einfache Spezialfälle berechnet hatten, wurde von $\mathrm{H} \mathrm{e} \mathrm{rz} \mathrm{o} \mathrm{g}{ }^{3}$ die allgemeine geometrisch-optische Darstellung angegeben, die sich als besonders fruchtbar erwies. Es zeigte sich jedoch, daß in vielen Fällen die Vernachlässigung des Streufeldes eine zu einschneidende Ver-

* D 77 (Teilergebnis).

1 J. C l a s s e n, Phys. Z. 9, 762 [1908].

2 W. E. S t e p h e n s, Phys. Rev. 45, 513 [1934].

3 R. H e r z o g, Z. Phys. 89, 447 [1934].

4 R. H e rzog, Z. Phys. 97, 596 [1935].

5 N. D. C o g g e s h a l l, J. Appl. Physics 18, 855 [1947].

6 W. Ploch u. W. Walch er, P. Phys. 127, 274 [1950]. cinfachung darstellte, weshalb die Berücksichtigung desselben notwendig wurde. Dies ist bei Beschränkung auf Bahnen in der Symmetrieebene zwischen den Polschuhen auf zwei verschiedenen Wegen möglich. Erstens kann man Korrekturen an den bekannten Gleichungen anbringen, wodurch man aber automatisch die Kleinheit des Streufeldeinflusses voraussetzt. Dieser Weg wurde von $\mathrm{Herzog}{ }^{4}, \mathrm{Cog} g \mathrm{eshall}{ }^{5}$, $\mathrm{Ploch}$ und W a l c her ${ }^{6}$ und R e u t e r s wärd ${ }^{7}$ beschritten. Zweitens kann man aber auch für das gesamte abbildende Feld unter Einschluß des Streufeldes neue Abbildungsgleichungen aufstellen. Diesen Weg ging $\mathrm{S}$ i d a y ${ }^{8}$, der, an Arbeiten von $\mathrm{Cog}$ g e sh a $11^{9}$ anknüpfend, für das radialsymmetrische Feld die Abbildungsgleichung ableitete.

Man kann nun zeigen, daß stets für die Abbildung durch magnetische Sektorfelder eine formal der Newtonschen Abbildungsgleichung entsprechende Beziehung gilt, auch wenn

${ }^{7}$ C. R e u t e rswärd, Ark. Fys. 3, 53 [1951] ; 4, 159 [1951].

${ }^{8}$ R. E. S i d a y, Proc. Phys. Soc., Lold. 59, 905 [1947].

9 N. D. Cog g eshall u. M. M u skat, Phys. Rev. 66, 187 [1944]; N. D. Cogg e s h a 11 , Phys. Rev. 70, 270 [1946]. 